\title{
Efectividad del programa "Fortaleciendo el cuidado preventivo de enfermería" en el nivel de conocimientos, grado de cumplimiento de la valoración y medidas de control del riesgo de caídas en pacientes de los servicios de medicina del Hospital Base de la Red Asistencial Almenara, 2010.
}

\author{
Irene Mercedes Zapata-Silva \\ Unidad de Posgrado de Ciencias de la Salud, Escuela de Posgrado, Universidad Peruana Unión, \\ Alt. Km 19; Carretera Central, Chosica, Lima
}

\begin{abstract}
RESUMEN
Objetivo: Determinar la efectividad del programa "Fortaleciendo el cuidado preventivo de enfermería" en el nivel de conocimientos, grado de cumplimiento de la valoración y medidas de control del riesgo de caídas en pacientes de los servicios de Medicina. Hospital Base de la Red Asistencial Almenara. Material y Métodos: Estudio cuantitativo, explicativo, de diseño cuasi experimental con grupo de estudio y grupo control. La muestra fue de 80 enfermeras, 40 para cada grupo, seleccionadas por muestreo intencional. Durante la intervención se capacitó a las enfermeras, se aplicó la escala de John Downton y una guía de recomendaciones para evitar caídas. Los instrumentos fueron: un cuestionario y dos listas de cotejo, cuya confiabilidad se estableció mediante el Coeficiente alfa de Cronbach y el índice de Kappa de Cohen. Resultados: El nivel de conocimientos de las enfermeras del grupo de estudio se incrementó del $27.5 \%$ al 95\%, mientras que el grado de cumplimiento de la valoración del riesgo y medidas de control del riesgo de caídas, alcanzó el 55\% en el mismo grupo. Conclusiones: Se encontró diferencias estadísticamente significativas, según la prueba t de Student antes y después de la intervención, en las tres variables de estudio y entre el grupo de estudio y grupo control $(\mathrm{p}<0.01 \mathrm{y} \mathrm{p}<0.05)$, confirmando la hipótesis planteada.
\end{abstract}

Palabras clave: Intervención, cuidado preventivo de caídas, valoración y control del riesgo, adultos hospitalizados.

\begin{abstract}
Objective: To determine the effectiveness of the program "Strengthening nursing preventive care" in the level of knowledge of nurses, degree of compliance assessment and risk control measures for falls in patient's medical services. Base Hospital Almenara Healthcare Network. Materials and Methods: quantitative, explanatory, quasiexperimental design study group and control grou The sample consisted of 80 nurses, 40 for each group, selected by purposive sampling. During the intervention, nurses were trained, we applied the scale of John Downton, and a guide of recommendations to prevent falls. The instruments were: a questionnaire and two checklists whose reliability was established using Cronbach's alpha coefficient and Cohen's Kappa. Results: The level of knowledge of nurses in the study group increased from $27.5 \%$ to $95 \%$, while the degree of compliance with the risk assessment and risk control measures fall, reached $55 \%$ in the same grou Conclusions: We found statistically significant differences according to Student's t-test before and after the intervention in the three study variables between the study group and control group ( $\mathrm{p}<0.01$ and $\mathrm{p}<0.05)$, confirming the hypothesis.
\end{abstract}

Keywords: Intervention, fall preventive care, assessment and control of risk, hospitalized adults.

*Correspondencia: delicirenezapatas@yahoo.es, 51-1-6397347-51-1-958645391 


\section{INTRODUCCIÓN}

Uno de los eventos adversos frecuentes, en el cuidado hospitalario, son las caídas de pacientes durante la permanencia en el hospital; estas, representan un problema importante porque desencadenan una serie de trastornos funcionales y la pérdida de autonomía del paciente para satisfacer sus necesidades cotidianas (Juvé, Carbonell, Sánchez, Brossa, Ortí, y Villanueva, 1999).

En el Hospital Base de la Red Almenara el perfil del paciente, que experimenta más caídas, es la persona adulta mayor de sexo masculino, con limitación sensorial para ver o escuchar, marcha no estable, y que no llama para ser atendido, sufren la caída en horas de la madrugada entre las 01.00 y las 07.00 horas y en menor porcentaje, durante el día (Dpto. Enfermería Hospital Guillermo Almenara, 2006).

El paciente varón sufre la caída, mientras intentan ir al baño o alcanzar el urinario de su velador, en horas de la noche, donde hay menos iluminación; un número menor de pacientes caen mientras deambulan o están en la ducha, por lipotimia. Indistintamente de la causa de la caída, el 100\% requiere evaluación médica, el $85 \%$ requiere placas radiográficas y el $20 \%$ requiere tomografía; una sola caída puede representar la diferencia entre la vida y la muerte del paciente (Dpto. de Enfermería Hospital Nacional Guillermo Almenara, informes 2000-2005).

En Estados Unidos de Norteamérica, Gillespie, L., Gillespie, W., Robertson, M., Lamb, S., Cummings, R y Rowe, B. (2003) señalaron que las intervenciones realizadas en los centros asistenciales no han demostrado efectos contundentes en la reducción del problema, utilizando estrategias aisladas que es necesario, por tanto, asociar medidas sólidas de cuidado y evaluarlas de modo periódico.

Watson (citada por Marriner, 1996), sustenta que la enfermera, como responsable del cuidado directo del paciente y por tanto de proteger su integridad, necesita poseer las competencias indispensables para garantizar la valoración del riesgo de caídas y aplicar las medidas respectivas de cuidado para el control de forma permanente y eficaz

Estas consideraciones motivaron el interés para el desarrollo del presente estudio de intervención, con el objetivo de determinar la efectividad del programa "Fortaleciendo el cuidado preventivo de enfermería" en el nivel de conocimientos, grado de cumplimiento de la valoración y control del riesgo de caídas de pacientes en los servicios de medicina del Hospital Base de la Red Asistencial Almenara.
El estudio se centra en el potencial de una intervención múltiple dirigida a las enfermeras, orientada a despertar mayor sensibilidad frente al problema y a la visualización del poder o capacidad que tiene en sus manos, para así disminuir el riesgo y procurar la autonomía y bienestar que pierde la persona que experimenta una caída.

\section{MATERIAL Y MÉTODOS}

La investigación se realizó en el Hospital Base de la Red Asistencial Almenara, EsSalud, de Nivel III de atención; sito en Avenida Grau N. ${ }^{\circ} 800$, distrito de la Victoria, durante los meses de marzo a setiembre del 2010. Según el alcance de los resultados, fue un estudio explicativo de diseño cuasi experimental, con grupo de estudio, grupo control y mediciones antes y después de la intervención "Fortaleciendo el cuidado preventivo".

La muestra fue de 80 pacientes, 40 para el grupo de estudio y 40 para el grupo de control; seleccionados mediante muestreo no probabilístico de tipo intencional. Las variables de interés fueron tres: el nivel de conocimientos de las enfermeras acerca de las caídas, los factores extrínsecos e intrínsecos relacionados, la valoración y el control del riesgo. La segunda variable fue el grado de cumplimiento de la valoración del riesgo, y la tercera fue el grado de cumplimiento de las medidas de control del riesgo aplicadas por las enfermeras, las mismas que se midieron en el grupo de estudio antes y después de la intervención y entre el grupo de estudio y de control, también antes y después de la intervención.

El programa "Fortaleciendo el cuidado preventivo de caídas" consistió en tres aspectos fundamentales: La capacitación de las enfermeras mediante cinco módulos de enseñanza realizados 4 veces por mes durante 3 meses, en tres grupos de enfermeras y talleres de autoestima y liderazgo en el cuidado; la aplicación de la Guía de Recomendaciones para evitar caídas (Arroyo del Cordero y Victoria Ochoa, 2005); la aplicación de la escala de valoración del riesgo de caídas de John Downton modificada y el uso de un dispositivo de alerta para pacientes de alto riesgo, durante siete meses del estudio.

Los instrumentos de recolección de datos fueron un cuestionario de tipo autoadministrado, con un total de 30 preguntas y cuya consistencia interna se determinó por el Coeficiente alfa de Cronbach $(0,91)$. La primera lista de cotejo que midió el grado de cumplimiento de la valoración del riesgo de caídas, que tuvo 20 ítemes. La segunda lista de cotejo midió el grado de cumplimiento de las medidas de control 
del riesgo de caídas, tuvo 24 ítemes. La confiabilidad de las listas de cotejo se estableció mediante el índice de Kappa de Cohen con valores de 0,7 para el grado de cumplimiento de la valoración del riesgo y 0,6 para el grado de cumplimiento de las medidas de control del riesgo de caídas.
El procesamiento y análisis de la información se realizó mediante el Paquete Estadístico para las Ciencias Sociales SPSS, versión 15,0 y el análisis inferencial, para comprobar las hipótesis, se realizó mediante la prueba $\mathrm{t}$ de Student para muestras independientes y para muestras relacionadas.

\section{RESULTADOS}

Tabla 1

Enfermeras de los grupos de estudio y control del Hospital Base de la Red Asistencial Almenara según nivel de conocimientos sobre la valoración y medidas de control del riesgo de caídas antes y después de la intervención "Fortaleciendo el cuidado preventivo de Enfermería”, 2010.

\begin{tabular}{ccccccccc}
\hline \multirow{2}{*}{ Conocimientos } & \multicolumn{4}{c}{ Antes } & \multicolumn{5}{c}{ Después } \\
& \multicolumn{2}{c}{ Grupo estudio } & \multicolumn{2}{c}{ Grupo control } & \multicolumn{2}{c}{ Grupo estudio } & \multicolumn{2}{c}{ Grupo control } \\
\cline { 2 - 10 } & $\mathrm{N}^{\circ}$ & $\%$ & $\mathrm{~N}^{\circ}$ & $\%$ & $\mathrm{~N}^{\circ}$ & $\%$ & $\mathrm{~N}^{\circ}$ & $\%$ \\
\hline Excelente & 11 & 27.5 & 0 & 0.0 & 38 & 95.0 & 12 & 30.0 \\
Bueno & 13 & 32.5 & 0 & 0.0 & 1 & 2.5 & 19 & 47.5 \\
Regular & 15 & 37.5 & 2 & 5.0 & 1 & 2.5 & 6 & 15.0 \\
Malo & 1 & 2.5 & 18 & 45.0 & 0 & 0.0 & 3 & 7.5 \\
Muy Malo & 0 & 0.0 & 20 & 50.0 & 0 & 0.0 & 0 & 0.0 \\
\hline Total & 40 & 100.0 & 40 & 100.0 & 40 & 100.0 & 40 & 100.0
\end{tabular}

En la tabla 1 se evidencia el incremento del nivel de conocimientos óptimo en el grupo de estudio, del $27.5 \%$ al 95\%, después de la intervención; mientras en el grupo control, el incremento es del $30 \%$. El nivel regular de conocimientos en el grupo de estudio se reduce de $37.5 \%$ a $2.5 \%$ y el nivel malo y muy malo desaparece en este grupo.

Tabla 2

Enfermeras de los grupos de estudio y control del Hospital Base de la Red Asistencial Almenara, según el grado de cumplimiento de la valoración del riesgo de caídas antes y después de la intervención "Fortaleciendo el cuidado preventivo de enfermería", 2010.

\begin{tabular}{ccccccccc}
\hline \multirow{2}{*}{$\begin{array}{c}\text { Grado de } \\
\text { cumplimiento }\end{array}$} & \multicolumn{3}{c}{ Antes } & \multicolumn{4}{c}{ Después } \\
\cline { 2 - 9 } & \multicolumn{2}{c}{ Grupo estudio } & \multicolumn{2}{c}{ Grupo control } & \multicolumn{2}{c}{ Grupo estudio } & \multicolumn{2}{c}{ Grupo control } \\
\cline { 2 - 9 } & $\mathrm{N}^{\circ}$ & $\%$ & $\mathrm{~N}^{\circ}$ & $\%$ & $\mathrm{~N}^{\circ}$ & $\%$ & $\mathrm{~N}^{\circ}$ & $\%$ \\
\hline Óptimo & 0 & 0 & 0 & 0 & 22 & 55.0 & 0 & 0.0 \\
Bueno & 9 & 22.5 & 13 & 32.5 & 10 & 25.0 & 11 & 27.5 \\
Regular & 13 & 32.5 & 9 & 22.5 & 5 & 12.5 & 14 & 35.0 \\
Malo & 18 & 45 & 18 & 45 & 3 & 7.5 & 15 & 37.5 \\
\hline Total & 40 & 100 & 40 & 100 & 40 & $100 \%$ & 40 & $100 \%$
\end{tabular}

En la tabla 2 se observa que después de la intervención, en el grupo de estudio, el grado de cumplimiento de la valoración del riesgo de caídas en el nivel óptimo no existe, pasando al 55\% después de la intervención. En este mismo grupo el grado de cumplimiento regular y malo, se reducen a $12.5 \%$ y $7.5 \%$, respectivamente. En el grupo de control la variación en los porcentajes es menor y el nivel óptimo de cumplimiento de la valoración del riesgo de caídas, no existe ni antes ni después. 
Tabla 3

Enfermeras de los grupos de estudio y control del Hospital Base de la Red Asistencial Almenara según el grado de cumplimiento de las medidas de control del riesgo de caídas antes y después de la intervención "Fortaleciendo el cuidado preventivo de enfermería”, 2010.

\begin{tabular}{ccccccccc}
\hline $\begin{array}{c}\text { Grado de } \\
\text { cumplimiento } \\
\text { de medidas }\end{array}$ & \multicolumn{3}{c}{ Antes } \\
\cline { 2 - 11 } & \multicolumn{2}{c}{ Grupo estudio } & \multicolumn{2}{c}{ Grupo control } & \multicolumn{2}{c}{ Grupo estudio } & Grupo control \\
& $\mathrm{N}^{\circ}$ & $\%$ & $\mathrm{~N}^{\circ}$ & $\%$ & $\mathrm{~N}^{\circ}$ & $\%$ & $\mathrm{~N}^{\circ}$ & $\%$ \\
\hline Óptimo & 0 & 0.0 & 0 & 0 & 22 & 55.0 & 0 & 0.0 \\
Bueno & 8 & 20.0 & 0 & 0 & 13 & 32.5 & 1 & 2.5 \\
Regular & 17 & 42.5 & 1 & 2.5 & 1 & 2.5 & 23 & 57.5 \\
Malo & 14 & 35.0 & 38 & 95.0 & 4 & 10.0 & 16 & 40.0 \\
Muy Malo & 1 & 2.5 & 1 & 2.5 & 0 & 0.0 & 0 & 0.0 \\
\hline Total & 40 & 100 & 40 & 100 & 40 & 100 & 40 & 100
\end{tabular}

En la tabla 3 se puede apreciar igualmente que, después de la intervención, el grado de cumplimiento de las medidas de control del riesgo de caídas varía en el grupo de estudio, del $0 \%$ al $55 \%$; disminuye notablemente en el nivel regular, del $42,5 \%$ al $2.5 \%$ y de la misma manera en los niveles malo y muy malo. En el grupo de control, sin embargo, el grado de cumplimiento no se incrementa en el nivel bueno y se mantiene alto en los niveles regular y malo.

\section{DISCUSIÓN}

En el presente estudio, el incremento registrado en el nivel óptimo de conocimientos sobre valoración y medidas de control del riesgo de caídas, se evidencia la efectividad del programa de intervención "Fortaleciendo el cuidado preventivo", con las enfermeras del grupo de estudio. Los hallazgos manifiestan que las profesionales participantes, cuentan con las competencias necesarias para un adecuado control del riesgo asistencial de caídas en los pacientes adultos hospitalizados. El incremento de conocimientos se verificó en las dimensiones de la identificación del riesgo, los factores intrínsecos y extrínsecos que subyacen en la causalidad de las caídas, la importancia de contar con instrumentos que permitan cuantificar el riesgo y, por lo tanto, aplicar las medidas destinadas a evitar eventos previsibles en el proceso de cuidados de la enfermera, mediante acciones dirigidas al paciente, al entorno y a la familia durante la hospitalización.

En consonancia con la literatura revisada, los estudios sobre el riesgo y el control de las caídas en el ambiente hospitalario se han limitado al establecimiento de la prevalencia del problema en adultos mayores y a la profundización sobre los factores de riesgo. No obstante, Gillespie M., Gillespie, W., Robertson, Lamb, Cummings, y Rowe (2003), coincidentemente con los resultados de la investigación realizada, señalan que es necesario valorar el papel de las intervenciones múltiples en materia de prevención de caídas, porque aun cuando los efectos protectores han demostrado un margen pequeño de beneficios, el conocimiento generado entre el personal de salud y la adecuación al contexto organizativo en que se producen las caídas, es suficiente para impulsar el compromiso para la adopción de prácticas seguras.

Vinculado al nivel de conocimientos, sobre los factores de riesgo y las acciones en materia preventiva de caídas, están los hallazgos de Ferrer Herrera, Jiménez, Hernández, Ferrer Tan y Llanes (2007), cuya intervención educativa se dirige a pacientes y familiares y no a enfermeras. No obstante, guarda similitudes con la investigación efectuada, en el incremento de conocimientos de algunos factores intrínsecos, entre ellos: la pérdida de la agudeza visual en la ocurrencia de caídas que llegó al 82.7\%, la existencia de mayor vulnerabilidad del paciente cuando hay cambios músculo esqueléticos y marcha inestable en un 78.8\%; los familiares lograron el $100 \%$ de incremento en el nivel de conocimientos sobre la importancia de la iluminación, el orden de los ambientes y la higiene de los pisos, en la ocurrencia de las caídas. En el estudio realizado, luego de la aplicación del programa de intervención "Fortaleciendo el cuidado preventivo" se logró que del $27.5 \%$ de conocimientos que presentaban las enfermeras del grupo de estudio en el nivel excelente, se incrementara al $95 \%$ en el mismo nivel.

Con referencia al grado de cumplimiento de la valoración del riesgo de caídas en pacientes hospitalizados, el incremento significativo registrado en el grado óptimo (55\%), evidencia que el programa 
de sensibilización realizado y la aplicación de la escala de John Downton modificada, conduce a un mayor compromiso de la enfermera al aplicar sus conocimientos y destrezas en la utilización de recursos tecnológicos apropiados, de bajo costo y beneficio significativo para los pacientes.

Un aporte específico del presente estudio radica en que la escala original de Downton no incluye la edad ni la incontinencia como factores de riesgo de caída, no obstante, en base a las evidencias disponibles (Corcuera, Ormeño y Zapata, 2008), y recomendaciones específicas de la Comisión Nacional de Arbitraje Médico (2008), se adicionó ambos criterios, innovando el instrumento y dotándolo de mayor funcionalidad para fines de control del riesgo.

Acerca de la utilización de fármacos, como criterio de valoración del riesgo de caídas en la escala de John Downton modificada, Passaro, Volpato, Romagnomi, Manzoli, y Zuliani (2000), avalan la necesidad de incluir este factor; precisa que aun siendo el $2.2 \%$ de pacientes de un total de $34.4 \%$ quienes ingieren benzodiacepinas y sufren riesgo de caídas. Es necesario considerar que un factor de riesgo cualquiera, se asocia a diversos factores personales, ambientales $\mathrm{u}$ organizativos, aumentando el potencial de caídas del paciente hospitalizado; motivo por el cual este criterio se mantuvo sin modificación alguna.

Del mismo modo, guardan similitud a favor del criterio del uso de fármacos como factor de riesgo de caídas, los hallazgos de Leipzig (1999 citado por André da Silva y Gómez, 2008), quienes encontraron una pequeña pero consistente asociación entre las caídas y la mayoría de los fármacos psicotrópicos $(\mathrm{OR}=1,73$ $\mathrm{IC}=95 \%)$.

Cabe destacar que las diferencias estadísticamente significativas encontradas en el grado de cumplimiento de la valoración del riesgo de caídas, según la escala de Downton en el grupo de estudio después de la intervención y entre este y el grupo control, se ha fortalecido con el uso de la alerta diseñada para facilitar la comunicación entre el personal de los diferentes turnos, al focalizar la atención del personal en pacientes de mayor riesgo.

Esta medida complementaria al uso de la escala de valoración es un dispositivo recomendado por Tena (2010). El uso del dispositivo de alerta en la intervención realizada, facilita una permanente vigilancia compartida entre los responsables del cuidado, que beneficia al paciente en orden a prevenir la caída. Se han utilizado y utilizan alertas para diversos riesgos, sin embargo, para la prevención de caídas constituye una innovación del presente estudio, con la ventaja de sintetizar en la frase "Protégeme", un mensaje del contenido del Módulo III de la etapa de capacitación titulado: Cuidar es proteger.

Watson (citada por Marriner, 1996) argumenta y promueve la reflexión sobre la responsabilidad moral y ética de la enfermera de proteger al paciente, de toda forma de riesgos que vulneren su autonomía y dignidad; fundamentada en la filosofía del cuidado y en dos íconos bíblicos que sustentan los valores humanos y cristianos frente a la persona enferma: la parábola del Buen Pastor y la parábola del Buen Samaritano. Después de la intervención, las enfermeras mostraron un grado óptimo de cumplimiento de las medidas preventivas de caídas, hasta en un 55\%. El incremento en el nivel de conocimientos fue mayor que en la práctica de la valoración y medidas de control del riesgo de caídas, situación que deja entrever la necesidad de seguir consolidando las mejoras implementadas.

Es preciso mencionar que no solo se logró comprobar la efectividad de la intervención, sino que mejoró notablemente la sensibilidad evidenciada en el modo cómo asumen, las enfermeras, la dimensión ético-científica de su profesión además de alcanzar instrumentos concretos que posibilitan una permanente verificación de la eficacia del proceso de valoración y de la oportuna toma de decisiones de intervención de las enfermeras, en el campo preventivo de caídas, uno de los efectos adversos más frecuentes y de consecuencias funestas para el paciente, el personal y la propia institución prestadora de servicios.

\section{CONCLUSIONES}

La aplicación del programa "Fortaleciendo el cuidado preventivo de enfermería" fue efectiva al obtener puntuaciones medias más altas en el nivel de conocimientos, grado de cumplimiento de la valoración y medidas de control del riesgo de caídas en pacientes adultos hospitalizados de los servicios de Medicina del Hospital Base de la Red Asistencial Almenara, tanto en el grupo de estudio antes y después de la intervención, como entre el grupo de estudio y el grupo de control. Estadísticamente significativo. $(\mathrm{p}<0,01)$. 


\section{REFERENCIAS BIBLIOGRÁFICAS}

André da Silva, Z y Gómez, A. (2007). Factores de riesgo de caídas en ancianos: revisión sistemática. En: Rev Saude Pública 2008; 42(5):946-56. Disponible on line en: www.scielo.br/pdf/rsp/ v42n5/6793. Recuperado el 12 -12-2010.

Arroyo del Cordero y Victoria Ochoa. (2005). Recomendaciones específicas para enfermería en la prevención de caídas. En: Desarrollo científico de enfermería 14, 145-150. España: Editorial Corporativo en medios de

Corcuera, L., Ormeño, R y Zapata, I. (2008). Incidencia de caídas en pacientes hospitalizados. Factores relacionados, Lima: Hospital Nacional Guillermo Almenara I. Es salud.

Perú, Departamento de Enfermería Hospital Nacional Guillermo Almenara. (2000 - 2005 - 2006). Informes estadísticos mensuales. Lima: Autor.

Ferrer Herrera, Jiménez, Hernández, Ferrer Tan y Llanes. (2007). Intervención educativa en ancianos de una casa de reposo sobre caídas en el hogar. En: Rev. Archivo Médico de Camagüey, versión on line V.12n6 nov-dic. 2008. ISSN 1025-0255.Disponible: www.Scielo.sld.cu.scielo.ph Recuperado el 2611-2010.

Gillespie, L., Gillespie, W., Robertson, M., Lamb, S., Cummings, R y Rowe, B. (2003). Intervenciones para la prevención de caídas en las personas ancianas USA: Grupo Cochrane.
Juvé, M., Carbonell, M., Sánchez, P., Brossa, M., Ortí, F y Villanueva, M. $(1,999)$. Riesgo de caídas en pacientes hospitalizados. Enfermería Clínica: 9, 257-263. Barcelona: Ed. Doyma.

Marriner, T. (1996). Modelos y teorías de enfermería ( $3^{\mathrm{a}}$ ed.) Madrid: Editorial Mosby.

Passaro A., Volpato, S., Romagnomi, F., Manzoli, N y Zuliani, G. (2000). Benzodiazepines with Different Hall-life and Falling in a Hospitalized Pulation: The Gifa study. Journal of Clinical Epidemiology. $53,222-1229$.

Tapia, M., Salazar, C., Tapia Colex, L y Olivares, E. (2002). Prevención de Caídas, indicador de calidad en el cuidado enfermero. Enfermería Cardiológica 11: 51-57.

Tena Tamayo, C., Ochoa, R., Sánchez, J., Manuell, G., Ramírez, A., Alvear, C., Casa, O., Guzmán, R. et al (2006). Recomendaciones específicas para enfermería relacionadas con la prevención de caída de pacientes durante la hospitalización. En Rev CONAMED. V11n5 enero-marzo 2006. Disponible en: www.salud.gob.mx/unidades/cdi/ DOCSAL7587.pdf. Recuperado el 7-I-2010.

Waldow Vera Regina. (2004/2008). Cuidar: expresión humanizadora. Reimpresión. Brasil: Editorial Palabra. 